from the physician's role in a specific clinical program to his/ her association with the parent clinical department. Thus, it would be crucial to ensure that the necessary supporting resources and infrastructure are in place, e.g. robust information systems that can measure, collect, analyse and report data in an accurate and timely manner, which would coincide with health organisations' increasing focus on the use of information systems in delivering effective value-based healthcare.

\section{Communication with patients}

\section{TAKEPHONERSHIP}

Scott Deacon*, David Wynne-Jones. Bristol Dental Hospital, University Hospitals Bristol NHS Foundation Trust

\subsection{6/leader-2018-FMLM.58}

Issue Our inability to answer the phone and resolve patient problems in a timely manner. This manifested as:

- Poor patient experience

- Answerphone messages not actioned

- Incorrect diverting of calls

- Unnecessary emails between teams

- Lack of education around the role of admin teams.

Bristol Dental Hospital Executive commissioned work to resolve this which had clinical leadership, Consultant Scott Deacon and managerial leadership, Deputy Divisional Director David Wynne-Jones.

Strategy for improvement A task and finish group was setup including clinicians and admin stakeholders.Additional drop in sessions were setup to engage staff and embed a culture of ownership.The initiative was called 'TakePhonership' and the Trust communications team supported creating the brand.

The initiative went live with data to highlight problem areas.Drop in sessions were well attended and straightforward actions were implemented.Including:

- Check telephone number accuracy on website and letters

- A 'Power Hour' - focussed on the busiest call times

- Purchasing headsets

- Educating departments on team roles

- Embed culture of responsibility

- Looking at various call routing mechanisms

- Engaging with centralised call centre

- Issuing anonymised updates

The agreed measurements of improvement were:

- Volume of calls

-\% of overall calls answered

$-\%$ of overall calls answered when available

- Level of telephone related complaints

These are reviewed and fed back to teams via email, meetings, and ongoing engagement drop in sessions and leadership walk rounds.

Measurement We now receive under 3000 calls a week; almost halving the call volume.At the height of complaints only $66 \%$ of calls were being answered when we were available. This has risen to over $75 \%$ and is likely to exceed $80 \%$. Complaints have fallen significantly by $300 \%$.

Impact Bristol Dental Hospital has taken 'phonership' and changed the culture is. This initiative has been adopted across the Trust.

\section{Leading across complex systems and organisation}

\section{IMPROVING IV FLUID PRESCRIPTION IN NEWCASTLE, UK - A TRAINEE DOCTOR LED QUALITY IMPROVEMENT PROJECT}

Robert Samuel*, Phil Laws. Newcastle upon Tyne NHS Foundation Trust, UK

10.1136/leader-2018-FMLM.59

Aims The aim was to improve the prescription of intravenous (IV) fluids in the emergency department, medical wards and surgical wards using principles of leadership and service improvement.

Methods The proposed change to practice was to introduce a new IV fluid prescription sheet that contained decision-aids that closely matched the most recent NICE guidelines on IV prescription in adults. The change was assessed in three ways. Firstly, the NICE clinical audit tool for IV fluid prescription in adults was performed before and during the pilot period by junior doctors working in the acute medical unit (AMU). Secondly, electronic records for sodium and potassium blood results were measured for the first 7 days of admission both before and during the intervention. Chi-square test was used to test for significance. Thirdly, a feedback sheet was put up in the nursing and non-medical staff room for comments on the intervention. Principles of trainee doctor leadership such as early senior medical staff buy-in, multi-disciplinary involvement and qualitative feedback were used.

Results There was a statistically significant reduction in rates of hypokalaemia, defined as a potassium result below the reference range, for the first 7 days following admission to hospital via the AMU. There was a mixed response to the NICE Clinical Audit tool, with improvements in some areas and reductions in others. Qualitative feedback helped discover issues with the sheet not previously identified.

Conclusions It is possible to lead a service improvement project as a foundation trainee. The principles of medical leadership and service improvement genuinely help to make small ideas into reality. This project shows that small simple changes to frequently performed tasks in a hospital have the potential to impact patient safety. The trial fluid prescription sheet is being used to inform the implementation and design of electronic IV fluid prescribing within the trust.

\section{Developing effective leaders}

\section{THE ACADEMIC FOUNDATION PROGRAMME IN HEALTHCARE LEADERSHIP AND MANAGEMENT - RECOMMENDATIONS BASED ON TRAINEE EXPERIENCE}

Natasha Szmidt, Charlotte Stanley*, Steven Tran. Torbay and South Devon NHS Trust, UK

10.1136/leader-2018-FMLM.60

The Academic Foundation Programme (AFP) provides themed posts in leadership and management; since 2015 two posts a year have been provided by Torbay and South Devon NHS Foundation Trust (TSDFT). Early leadership development in trainees is recognised as important ${ }^{1}{ }^{2}$ but a lack of leadership opportunities for junior doctors remains. ${ }^{2}$ The program at 